\title{
Effects of a Parallel Magnetic Field on the Metal-Insulator Transition in a Dilute Two-Dimensional Electron System
}

\author{
Kevin Eng ${ }^{1,2}$, X. G. Feng ${ }^{1, *}$, Dragana Popović ${ }^{1}$, and S. Washburn ${ }^{2}$ \\ ${ }^{1}$ National High Magnetic Field Laboratory, Florida State University, Tallahassee, FL 32310 \\ ${ }^{2}$ Department of Physics and Astronomy, The University of North Carolina at Chapel Hill, Chapel Hill, NC 27599
}

(November 19, 2018)

\begin{abstract}
The temperature dependence of conductivity $\sigma(T)$ of a two-dimensional electron system in silicon has been studied in parallel magnetic fields $B$. At $B=0$, the system displays a metal-insulator transition at a critical electron density $n_{c}(0)$, and $d \sigma / d T>0$ in the metallic phase. At low fields $(B \lesssim 2 \mathrm{~T}), n_{c}$ increases as $n_{c}(B)-n_{c}(0) \propto B^{\beta}(\beta \sim 1)$, and the zero-temperature conductivity scales as $\sigma\left(n_{s}, B, T=0\right) / \sigma\left(n_{s}, 0,0\right)=f\left(B^{\beta} / \delta_{n}\right)$ (where $\delta_{n}=\left(n_{s}-n_{c}(0)\right) / n_{c}(0)$, and $n_{s}$ is electron density) as expected for a quantum phase transition. The metallic phase persists in fields of up to $18 \mathrm{~T}$, consistent with the saturation of $n_{c}$ at high fields.
\end{abstract}

PACS Nos. 71.30.+h, 71.27.+a, 73.40.Qv

The possibility of a metal-insulator transition (MIT) in two dimensions (2D) has been a subject of intensive research in recent years [1,2]. Most studies have focused on the conventional metallic behavior where the conductivity $\sigma$ increases with decreasing temperature $T$ (i. e. $d \sigma / d T<0)$ but the origin of such $\sigma(T)$ is still not understood. Similarly, a dramatic decrease of $\sigma$ with magnetic fields $B$ applied parallel to the $2 \mathrm{D}$ plane remains puzzling. Above some characteristic field, which is a function of the carrier density $n_{s}$, this unexpectedly large negative magnetoconductance is followed by a weaker dependence on $B$. Parallel $B$ has been also shown to suppress the metallic $T$-dependence $(d \sigma / d T<0)$ [3 5 and transform it into an insulatinglike behavior $(d \sigma / d T>0)$. Since it has been commonly assumed that $d \sigma / d T>0$ is necessarily related to an insulating state at $T=0$, this was taken as evidence that high parallel $B$ eliminates the MIT and the 2D metallic phase entirely [1]. The field where $d \sigma / d T$ changes sign (for a fixed $n_{s}$ ) has been identified as the critical field for a field-induced MIT [5]. Furthermore, it has been established that this critical field is comparable both to the field where the crossover from a low-field to high-field regime takes place and to the field where $2 \mathrm{D}$ carriers become fully spin-polarized [4, 6 , 8], suggesting that the 2D metallic phase may exist only in a spinunpolarized system. By contrast, the methods that do not use the sign change of $d \sigma / d T$ as a criterion to determine $n_{c}$ have yielded [9] finite values of $n_{c}$ even in high parallel $B$ where $d \sigma / d T>0$ at all $n_{s}$, which implies that the 2D metal may exist even for spinless electrons. It is clear that the fate of the metallic phase in a parallel $B$ represents one of the major open issues in the studies of dilute, strongly interacting 2D systems.

The main difficulty associated with the attempts to resolve this issue so far [3 9 has been that $\sigma(T)$ does not have a simple form for $n_{s}>n_{c}(0)$ in parallel $B$. Therefore, this does not allow one to make reliable extrapolations to $T=0$ and to establish the existence of a true
MIT with high credibility. In addition, there is no theoretical justification 10 for assuming that the $2 \mathrm{D}$ metallic phase can be characterized only by $d \sigma / d T<0$. In disordered 3D metals, for example, it is well known that this derivative can be either negative or positive near the MIT [11]. More relevant to this work, a recent study [2] of a $2 \mathrm{D}$ electron system in $\mathrm{Si}$ metal-oxide-semiconductor field-effect transistors (MOSFETs) in $B=0$ has established unambiguously that the metallic phase may be described by $d \sigma / d T>0$ such that $\sigma$ decreases to a nonzero value as $T \rightarrow 0$. This occurs when some of the localized states in the tail of the upper electric subband 12 also become populated [14]15] as the subband splitting is reduced by applying voltage $\left(V_{\text {sub }}\right)$ to the Si substrate [13]. Such localized states act as additional scattering centers for $2 \mathrm{D}$ electrons [16], and perhaps may even act as local magnetic moments if they are singly occupied (due to a large on-site Coulomb repulsion) 15, 17.

Regardless of the detailed microscopic picture, the 2D metal with $d \sigma / d T>0$ in $B=0$ exhibits two features [2] that distinguish it from the $d \sigma / d T<0$ case, and that are of crucial importance for the study of $\sigma$ in parallel $B$. First, $\sigma(T)$ follows a simple and precise form over a very broad (two decades) range of $T$ : $\sigma\left(n_{s}, T\right)=\sigma\left(n_{s}, T=0\right)+C\left(n_{s}\right) T^{2}$. This allows a reliable extrapolation to $T=0$, which yields $\sigma\left(n_{s}, T=0\right) \sim \delta_{n}^{\mu}$ $(\mu \approx 3)$, as expected in the vicinity of the MIT [10,18]. Second, near the MIT, the data obey dynamical scaling $\sigma\left(n_{s}, T\right)=\sigma_{c}(T) f\left(T / \delta_{n}^{z \nu}\right)$ with $\sigma_{c}=\sigma\left(n_{s}=n_{c}, T\right) \sim T^{x}$ $(z \nu=1.3 \pm 0.1, x \approx 2.6, \mu=x(z \nu)=3.4 \pm 0.4)$, both in agreement with theoretical expectations near a quantum phase transition [10] and consistent with the extrapolations of $\sigma(T)$ to $T=0$. Here we show that such features allow us to establish a phase diagram in the $\left(\delta_{n}, B, T=0\right)$ plane. We find that the behavior of low-field $\sigma(B)$ is consistent with the phase diagram, and can be attributed to an increase in $n_{c}(B)$, as expected near a true MIT [10]. The saturation of $n_{c}(B)$ observed 


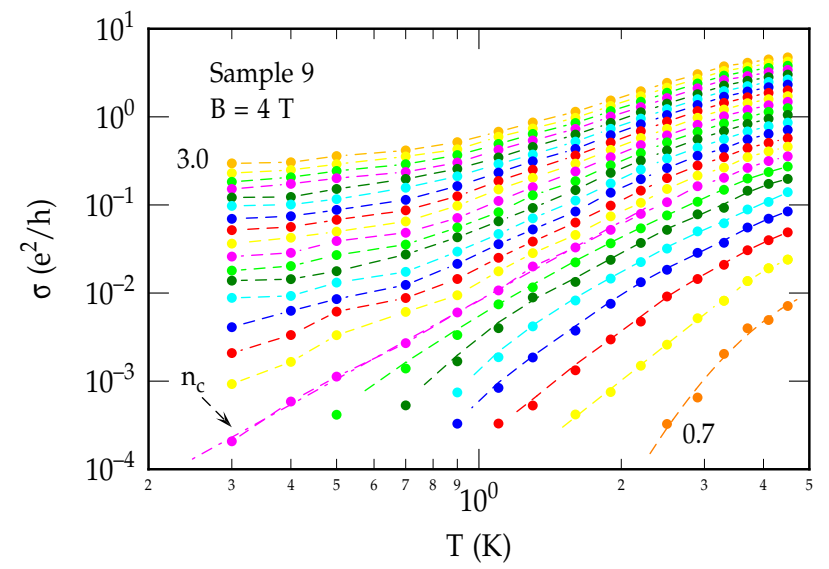

FIG. 1. $\sigma(T)$ for sample 9 at $B=4$ T. $n_{s}$ varies from $3.0 \times 10^{11} \mathrm{~cm}^{-2}$ (top) to $0.7 \times 10^{11} \mathrm{~cm}^{-2}$ (bottom) in steps of $0.1 \times 10^{11} \mathrm{~cm}^{-2}$. $n_{c}(B=4 \mathrm{~T})=1.4 \times 10^{-11} \mathrm{~cm}^{-2}$ and is marked by the arrow. The dashed lines guide the eye. $\sigma_{c}$ clearly follows a simple power-law dependence on $T: \sigma_{c} \propto T^{x}$.

at higher $B$ strongly suggests that the $2 \mathrm{D}$ metal may exist even for spinless electrons.

Measurements were carried out on n-channel Corbino shaped Si MOSFETs (channel length $=0.4 \mathrm{~mm}$, mean circumference $=8 \mathrm{~mm}$ ) with poly-Si gates, selfaligned ion-implanted contacts, and the peak mobility $\sim 1 \mathrm{~m}^{2} / \mathrm{Vs}$ at $4.2 \mathrm{~K}$. Other sample details have been given elsewhere [14,15,2]. $n_{s}$ was controlled by the gate voltage $V_{g} . V_{\text {sub }}=+1 \mathrm{~V}$ was applied to minimize the subband splitting and maximize the $T$-range (up to $\sim 2 \mathrm{~K})$ in which the metallic behavior with $d \sigma / d T>0$ is observed [15,2]. Conductance was measured as a function of $V_{g}$ using standard low-noise analog lock-in techniques at $\sim 17 \mathrm{~Hz}$ and a low-noise current preamplifier (the lead resistance was subtracted by the usual method). Excitation voltages were kept low enough to ensure that the conduction was Ohmic at the lowest $T$ (see Ref. [2] for more details). A systematic study of $\sigma\left(n_{s}, T\right)$ was done in a $\mathrm{He}^{3}$ cryostat in static parallel fields up to $9 \mathrm{~T}$ and for $0.25 \leq T \leq 4 \mathrm{~K}$. Measurements up to $18 \mathrm{~T}$ were carried out with the sample in the mixing chamber of a dilution refrigerator with a base $T \approx 0.020 \mathrm{~K}$.

Figure 1 shows $\sigma(T)$ for sample 9 (also studied in Ref. [2]) at $B=4 \mathrm{~T}$ for various $n_{s}$ in the vicinity of the MIT. The data are qualitatively similar to the $B=0$ case [2]. For example, at the lowest $n_{s}\left(i\right.$. e. $\left.n_{s}<n_{c}\right)$, $\sigma$ decreases exponentially with decreasing $T$, indicating an insulating state at $T=0$. In the metallic phase $\left(n_{s}>n_{c}\right), \sigma(T)$ is weaker and its curvature is the opposite from the one expected for an insulating state. It clearly extrapolates to a finite value as $T \rightarrow 0$, as discussed in more detail below. As shown in Fig. 1, $n_{c}$ is identified as the density where $\sigma_{c}=\sigma\left(n_{s}=n_{c}, T\right) \propto T^{x}$, consistent with the $B=0$ case and in agreement with general arguments [10]. The exponent $x$ is found to be

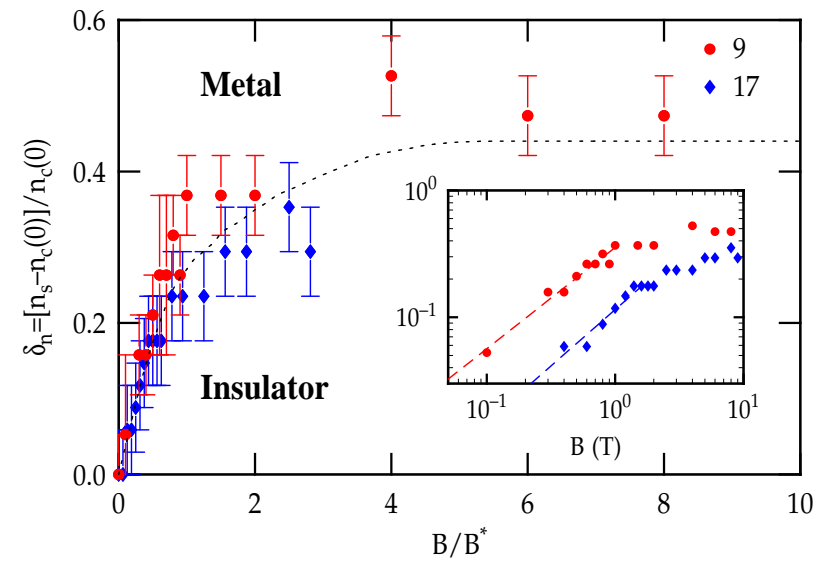

FIG. 2. $T=0$ phase diagram for two samples. The dashed line guides the eye. The boundary between metallic and insulating phases is described by a power-law relation (see inset) $\left[n_{c}(B)-n_{c}(0)\right] / n_{c}(0) \propto\left(B / B^{*}\right)^{\beta}$ at low fields, with the same crossover exponent $\beta \approx 0.9$ for both samples $\left(B^{*}=1 \mathrm{~T}\right.$ for sample 9$)$. Inset: the same data vs. $B$ on a log-log scale. The dashed lines are fits with the slopes equal to $\beta$. At $B=0$, $n_{c}\left(10^{11} \mathrm{~cm}^{-2}\right)=0.95 \pm 0.05$ and $0.85 \pm 0.05$ for samples 9 and 17 , respectively.

\section{$2.7 \pm 0.4$, and remains constant as a function of $B$.}

The critical density $n_{c}$ was determined in this way at each given $B$. Fig. 2 inset shows the relative change of $n_{c}(B)$ with respect to its zero-field value $n_{c}(0)$ as a function of $B$ for two different samples. At low fields $(B<2 \mathrm{~T}), n_{c}(B)$ increases with $B$ in a power-law fashion: $\left[n_{c}(B)-n_{c}(0)\right] / n_{c}(0) \propto B^{\beta}$, where the crossover exponent $\beta=0.80 \pm 0.06$ and $0.9 \pm 0.1$ for samples 9 and 17 , respectively. We point out that such a powerlaw shift of $n_{c}$ with $B$ is expected to occur in the case of a true MIT [10], and has been observed in several 3D systems 19]. In the case of conventional 2D metallic behavior in high-mobility Si MOSFETs, finite values of $n_{c}(B)$ were found based on vanishing activation energy and vanishing nonlinearity of current-voltage curves as extrapolated from the insulating side [9]. For those data, we find that the corresponding $\beta=1.1 \pm 0.1$. It is interesting that the crossover exponents $\beta$ are almost the same in both cases (Ref. [9] and Fig. 2) even though the $B=0$ behaviors of $d \sigma / d T$ are very different. Furthermore, in both cases the saturation of $n_{c}(B)$ is observed at higher fields. The determination of $n_{c}(B)$ based on a sign change of $d \sigma / d T$ has yielded $\beta \approx 1$ in high-mobility Si MOSFETs [ [] , and $\beta \approx 1.4$ in a $2 \mathrm{D}$ hole system in GaAs [5]. While the difference in $\beta$ between Si and GaAs devices might be due to the difference in the dominant scattering mechanisms in the two materials, it should be noted that the mere sign of $d \sigma / d T$ is clearly not a reliable indicator of the nature of the $(T=0)$ ground state.

Since $\beta \approx 0.9$ for both of our samples and only the absolute values of $n_{c}(B)$ are different, it is possible to create a generalized phase diagram in the $\left(\delta_{n}, B, T=\right.$ 
0) plane as shown in Fig. 2. The dashed line in Fig. 2 represents the boundary between metallic and insulating phases. It is clear that, for a fixed, small $\delta_{n}$, the system will undergo a magnetic field-driven MIT, leading to a strong suppression of magnetoconductance (MC). On the other hand, for densities far from $n_{c}(0)\left(i\right.$. e. large $\left.\delta_{n}\right)$, the $2 \mathrm{D}$ system will remain metallic with increasing $B$. In that case, one expects a much weaker MC. Exactly this kind of behavior of $\mathrm{MC}$ has been observed in both our samples [20] and high-mobility Si MOSFETs in the conventional metallic regime [6].

First we focus on the regime of low fields $(B \lesssim 2 \mathrm{~T})$ and small $\delta_{n}$, where MC exhibits a large drop [20]. We find that all the MC curves $\sigma(B) / \sigma(0)$ for a fixed $n_{s}$ and different $T$ can be collapsed onto one function using a single scaling parameter $\mathcal{F}\left(n_{s}, T\right)$ [Fig. 3(a)]. For each given $n_{s}$, we find empirically that $\mathcal{F}\left(n_{s}, T\right)=a\left(n_{s}\right)+b\left(n_{s}\right) T^{1.3 \pm 0.2}$ [Fig. 3(b)]. The parameter $a\left(n_{s}\right)$ decreases with decreasing $n_{s}$ and extrapolates to zero at a density equal to $(0.88 \pm 0.04) \times 10^{11} \mathrm{~cm}^{-2}$. Within our measurement error, this density is the same as $n_{c}(0)$. Fig. 3(a) inset shows that a fit to the critical form, $a\left(n_{s}\right)=a_{0} \delta_{n}^{1 / \beta}$, yields a crossover exponent $\beta=1.2 \pm 0.2\left(a_{0}=1.7 \pm 0.4\right)$, consistent with the value of $\beta$ that was established independently earlier (Fig. 2) based on the extrapolations of $\sigma(T)$ to $T=0$ in the metallic regime at fixed $B$ (Fig. 1). Moreover, Fig. 3(a) shows that it is also possible to collapse $\sigma(B) / \sigma(0)$ for all different $n_{s}$ and $T$ using a single scaling parameter $B_{0}\left(n_{s}, T\right)=\mathcal{A}\left(n_{s}\right) \mathcal{F}\left(n_{s}, T\right)$, which combines the previous scaling parameter $\mathcal{F}$ with a density dependent prefactor $\mathcal{A}\left(n_{s}\right)$. The fitting parameters $\mathcal{A}\left(n_{s}\right)=(2.8 \pm 0.3) n_{s}-(1.6 \pm 0.3)$ and $b\left(n_{s}\right)=$ $-(11.4 \pm 0.9) n_{s}+(14.9 \pm 0.9)$ (all $n_{s}$ in units of $10^{11} \mathrm{~cm}^{-2}$ ) do not show much variation near $n_{c}(0)$.

Therefore, our results demonstrate that the zerotemperature conductivity scales with $n_{s}$ and $B$ as

$$
\sigma\left(\delta_{n}, B, 0\right) / \sigma\left(\delta_{n}, 0,0\right)=f\left(B / \delta_{n}^{1 / \beta}\right),
$$

where $\beta \sim 1$. For a fixed $n_{s}$, the crossover function $f\left(B / \delta_{n}^{1 / \beta}\right)$ decreases by orders of magnitude with increasing $B$ [Fig. $3(\mathrm{a})$ ] as the system approaches the magnetic field-driven MIT. The critical fields $B_{c}$ for such a transition are given by $B_{c} \propto \delta_{n}^{1 / \beta}$ in agreement with the phase diagram determined earlier (Fig. 2). It is clear that the scaling parameter $B_{0}\left(n_{s}, T=0\right) \propto B_{c}$. We also note that the scaling plot in Fig. 3(a) includes a few densities below $n_{c}(0)$ in the quantum critical region, where $\mathcal{F}\left(n_{s}, T\right) \propto T^{1.3}$ in the given $T$ range. Furthermore, for $n_{s}=n_{c}(0)$, our results show that $\sigma(0, B, T) / \sigma(0,0, T)=f\left(B / T^{1.3}\right)$. According to standard scaling arguments [10], the exponent $1.3=\beta / z \nu$. With $\beta=1.2 \pm 0.2$, this yields $z \nu=0.9 \pm 0.3$, in reasonable agreement with $z \nu=1.3 \pm 0.1$ determined from scaling of $\sigma\left(n_{s}, T\right)$ in $B=0$ [2]. All these findings provide strong experimental evidence for a quantum phase
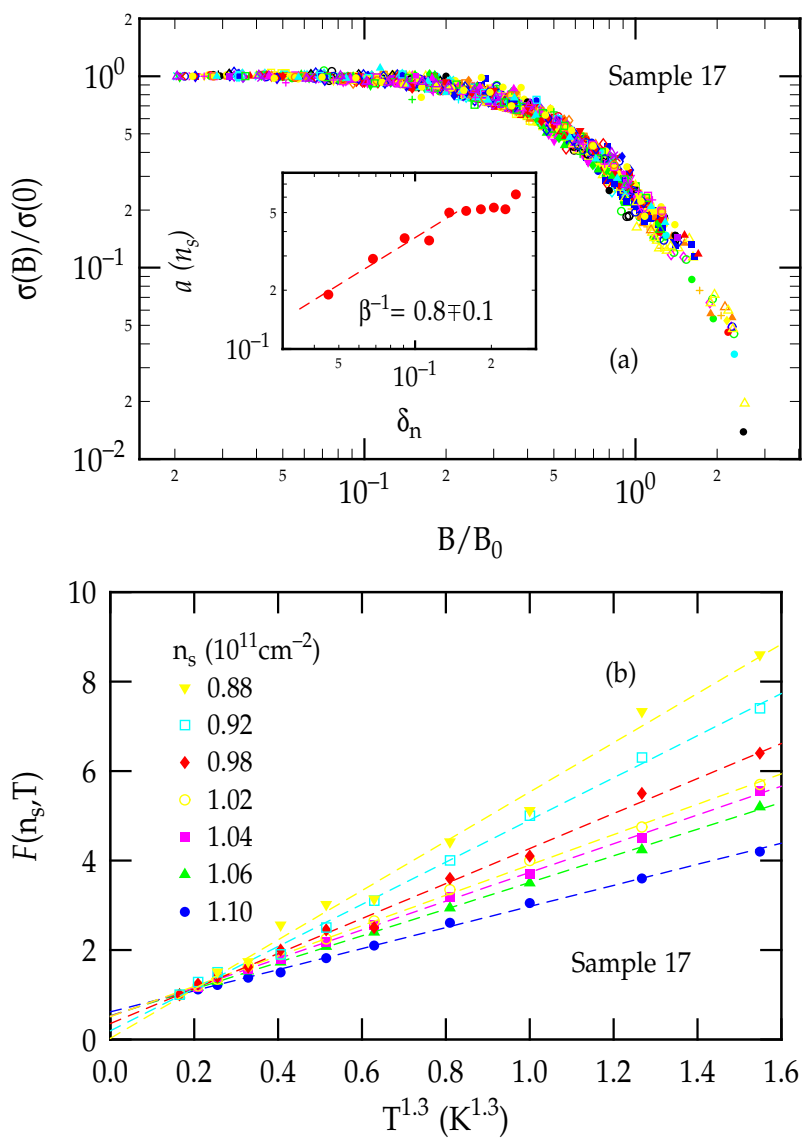

FIG. 3. (a) Scaling of low-field ( $B \lesssim 2 \mathrm{~T}$ ) magnetoconductivity for $n_{s}\left(10^{11} \mathrm{~cm}^{-2}\right)=0.80-1.10$ in steps of 0.02 , and ten different $T$ from 0.25 to $1.4 \mathrm{~K}$. The scaling parameter $B_{0}\left(n_{s}, T\right)=\mathcal{A}\left(n_{s}\right) \mathcal{F}\left(n_{s}, T\right)$. $\mathcal{F}\left(n_{s}, T\right)=a\left(n_{s}\right)+b\left(n_{s}\right) T^{1.3}$, as shown for several $n_{s}$. $a\left(n_{s}\right) \propto \delta_{n}^{1 / \beta}$, as shown in the inset of (a).

transition in both $B=0$ and $B \neq 0$ in our system.

Recent studies of some Si MOSFETs in the conventional metallic regime have shown 21] that, in that case, the $\mathrm{MC}$ curves can be also scaled with a single parameter $B_{\sigma}$, where $B_{\sigma}$ corresponds to the onset of full spin polarization of the electron system [22] $(\beta \approx 1.7$ and $\beta \approx 1$ in Refs. 21] and [22], respectively). However, since the form of $\sigma(T)$ in the conventional metallic regime does not allow reliable extrapolations to $T=0$ in $B \neq 0$, it is difficult to establish the existence of a metallic phase and $n_{c}(B)$ (or, equivalently, $B_{c}\left(n_{s}\right)$ ) with certainty. We showed earlier [14, 15] that our Si MOSFETs exhibit exactly the same kind of conventional metallic behavior in absence of scattering by local magnetic moments. Therefore, we expect that similar values of $B$ will be required for full spin polarization of our $2 \mathrm{D}$ system at comparable $n_{s}$, even when a small fraction of electrons occupies the tail of the upper subband. Our work demonstrates that, in spite of the qualitative differences in $\sigma(T)$ in the two regimes (with and without local magnetic moments), the effect of the parallel $B$ on $\sigma$ is remarkably 


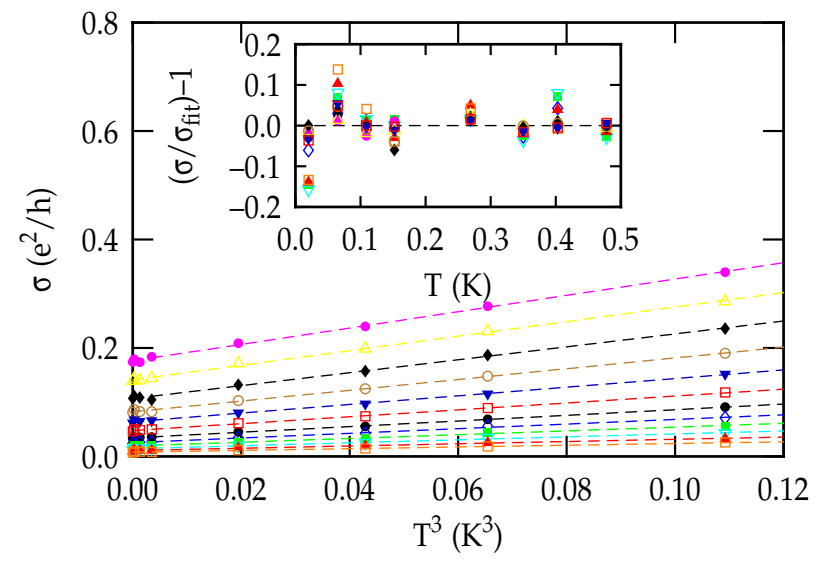

FIG. 4. Sample 19, $B=18 \mathrm{~T}: \sigma(T)$ plotted vs. $T^{3}$ for $n_{s}\left(10^{11} \mathrm{~cm}^{-2}\right)$ from 4.9 (top) to 3.8 (bottom) in steps of 0.1 ; $0.020 \lesssim T \leq 0.478 \mathrm{~K}$. The dashed lines are fits $\sigma_{f i t}\left(n_{s}, T\right)$. Inset: The deviation of the data from the fit $v s$. temperature.

similar. This strongly suggests that in our system at low fields $(B<2 \mathrm{~T})$, the critical fields $B_{c} \sim B_{\sigma}$, where $B_{\sigma}$ is the polarization field. Indeed, in that regime $B_{c}$ in Fig. 2 is comparable to $B_{\sigma}$ in Ref. 22] for the same $n_{s}$. In addition, since $B_{\sigma} \propto \delta_{n}$ continues to hold over a wide range of $n_{s}$ (up to $\delta_{n} \sim 10$ ) [22], this implies that for $\delta_{n} \sim(0.5-0.6)$ in Fig. 2, the 2D electrons will be fully spin polarized at $\sim 2 \mathrm{~T}$. Therefore, at $B>2 \mathrm{~T}$ the MIT will occur between a spin-polarized metal and a spin-polarized insulator. This conclusion is supported by our observation that $n_{c}$ no longer seems to depend on the magnetic field for $B>2 \mathrm{~T}$.

The detailed properties of the apparent spin-polarized $2 \mathrm{D}$ metallic phase and the concomitant MIT require further careful investigation that is beyond the scope of this Letter. Here we demonstrate only that the metallic phase persists even in fields as high as $18 \mathrm{~T}$, where we estimate 22] that the 2D system is fully spin polarized in the range of $n_{s}$ studied. We find that $\sigma(T)$ in the metallic phase follows a simple power-law form

$$
\sigma\left(n_{s}, B, T\right)=\sigma\left(n_{s}, B, T=0\right)+C\left(n_{s}, B\right) T^{\alpha(B)},
$$

similar to the $B=0$ case, where $\alpha(B=0)=2.0 \pm 0.1$ [2]. In both cases, finite values of $\sigma\left(n_{s}, B, T=0\right)$ indicate that the $2 \mathrm{D}$ system is in the metallic state. At $B=18 \mathrm{~T}$, however, $\sigma(T)$ is best described with $\alpha=3$ in Eq. (2), as shown in Fig. 1 . While the mechanism that leads to $\alpha=3$ is unclear, it is interesting that the $T^{3}$ term (albeit with a different sign) has been observed in the quasi$2 \mathrm{D}$ metallic $\mathrm{Sr}_{3} \mathrm{Ru}_{2} \mathrm{O}_{7}$ below $0.5 \mathrm{~K}$ near a metamagnetic quantum critical point [23]. In our case, such a jump in magnetization would occur as $2 \mathrm{D}$ electrons rearrange from an unpolarized to a spin-polarized metallic state with decreasing $n_{s}$ at a fixed high $B$. The $T^{3}$ variation of resistivity has been also observed in a low carrier density metallic state of $\mathrm{SrB}_{6}$ 24] but the microscopic mechanism is still not understood.

In summary, we have studied the effect of a parallel $B$ on the conductivity of a $2 \mathrm{D}$ electron system with $d \sigma / d T>0$ in the metallic phase, even at $B=0$. The zero-temperature $\left(\delta_{n}, B\right)$ phase diagram has been constructed based on the simple form of $\sigma(T)$ in the metallic regime. Furthermore, the low-field magnetoconductivity has been found to scale with $n_{s}$, consistent with the $\left(\delta_{n}, B, T=0\right)$ phase diagram. These findings provide strong experimental evidence for a quantum phase transition in both $B=0$ and $B \neq 0$. The metallic phase is found to persist in fields as high as $18 \mathrm{~T}$. Our results strongly suggest that the $2 \mathrm{D}$ metallic phase may exist even in the absence of spin degrees of freedom.

We are grateful to T. Murphy, E. Palm, and K. Walther for technical assistance. This work was supported by an NHMFL In-House Research Program grant, NSF Grants DMR-9796339 and DMR-0071668, NHMFL through NSF Cooperative Agreement DMR-9527035, and by ARO-EL-35059.

*Present address: Terayon Communication Systems, Santa Clara, CA 95054.

[1] See E. Abrahams, S. V. Kravchenko, and M. P. Sarachik, Rev. Mod. Phys. 73, 251 (2001), and references therein.

[2] X. G. Feng et al., Phys. Rev. Lett. 86, 2625 (2001).

[3] D. Simonian et al., Phys. Rev. Lett. 79, 2304 (1997); V. M. Pudalov et al., JETP Lett. 65, 932 (1997); M. Y. Simmons et al., Phys. Rev. Lett. 80, 1292 (1998); K. M. Mertes et al., Phys. Rev. B 63, 041101 (R) (2001).

[4] T. Okamoto et al., Phys. Rev. Lett. 82, 3875 (1999).

[5] J. Yoon et al., Phys. Rev. Lett. 84, 4421 (2000).

[6] S. A. Vitkalov et al., Phys. Rev. Lett. 85, 2164 (2000).

[7] E. Tutuc et al., Phys. Rev. Lett. 86, 2858 (2001).

[8] V. M. Pudalov et al., cond-mat/0103087 (2001).

[9] V. T. Dolgopolov et al., JETP Lett. 55, 733 (1992); M. R. Sakr et al., Phys. Rev. B 65, 041303(R), (2001); A. A. Shashkin et al., Phys. Rev. Lett. 87, 266402 (2001).

[10] D. Belitz et al., Rev. Mod. Phys. 66, 261 (1994).

[11] P. A. Lee and T. V. Ramakrishnan, Rev. Mod. Phys. 57, 287 (1985).

[12] Usually, this is a tail of the $E_{0^{\prime}}$ subband - the lowest subband associated with the four conduction band valleys with the light effective mass $\left(0.190 m_{e}\right)$ perpendicular to the interface. See also Ref. 13.

[13] See T. Ando, A. B. Fowler, and F. Stern, Rev. Mod. Phys. 54, 437 (1982), and references therein.

[14] D. Popović et al., Phys. Rev. Lett. 79, 1543 (1997).

[15] X. G. Feng et al., Phys. Rev. Lett. 83, 368 (1999).

[16] A. B. Fowler, Phys. Rev. Lett. 34, 15 (1975); A. Kastalsky and F. F. Fang, Surf. Sci. 113, 153 (1982); U. Kunze, J. Phys. C 17, 5677 (1984); S. Kawaji and N. Nagashima, Surf. Sci. 196, 316 (1988); D. Popović, F. F. Fang, and P. J. Stiles, Solid State Commun. 68, 25 (1988).

[17] For brevity, here we refer to this type of scattering as "local magnetic moments".

[18] N. Goldenfeld, Lectures on Phase Transitions and 
the Renormalization Group (Addison-Wesley, Reading, 1992).

[19] T. F. Rosenbaum et al., Europhys. Lett. 10, 269 (1989);

S. Bogdanovich et al., Phys. Rev. B 55, 4215 (1997); M. P. Sarachik et al., Phys. Rev. B 58, 6692 (1998); M. Watanabe et al., Phys. Rev. B 60, 15817 (1999).

[20] K. Eng et al., in Proceedings of the 25th International Conference on the Physics of Semiconductors, edited by N. Miura and T. Ando, Springer Proceedings in Physics Vol. 87 (Springer, Berlin, 2001), p. 741; condmat/0012063.

[21] S. A. Vitkalov et al., Phys. Rev. Lett. 87, 086401 (2001).

[22] A. A. Shashkin et al., Phys. Rev. Lett. 87, 086801 (2001).

[23] S. A. Grigera et al., Science 294, 329 (2001).

[24] H. R. Ott et al., Z. Phys. B 102, 337 (1997). 